\title{
Heavily phosphorus doped germanium: Strong interaction of phosphorus with vacancies and impact of tin alloying on doping activation $\oplus^{\circ}$
}

Cite as: J. Appl. Phys. 125, 225703 (2019); https://doi.org/10.1063/1.5107503

Submitted: 29 April 2019 . Accepted: 22 May 2019 . Published Online: 10 June 2019

Anurag Vohra (D), Afrina Khanam (D), Jonatan Slotte (D), Ilja Makkonen (D), Geoffrey Pourtois, Clement Porret (D), Roger Loo (D), and Wilfried Vandervorst

COLLECTIONS

EP This paper was selected as an Editor's Pick
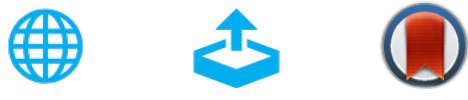

\section{ARTICLES YOU MAY BE INTERESTED IN}

Strategies and challenges of high-pressure methods applied to thermoelectric materials Journal of Applied Physics 125, 220901 (2019); https://doi.org/10.1063/1.5094166

Evolution of phosphorus-vacancy clusters in epitaxial germanium

Journal of Applied Physics 125, 025701 (2019); https://doi.org/10.1063/1.5054996

Computer modeling of single-layer nanocluster formation in a thin $\mathrm{SiO}_{2}$ layer buried in $\mathrm{Si}$ by ion mixing and thermal phase decomposition

Journal of Applied Physics 125, 225708 (2019); https://doi.org/10.1063/1.5096451

\section{Journal of Applied Physics Polymer-Grafted Nanoparticles}




\title{
Heavily phosphorus doped germanium: Strong interaction of phosphorus with vacancies and impact of tin alloying on doping activation
}

Cite as: J. Appl. Phys. 125, 225703 (2019); doi: 10.1063/1.5107503

Submitted: 29 April 2019 - Accepted: 22 May 2019.

Published Online: 10 June 2019

Anurag Vohra, ${ }^{1,2, a)}$ (D) Afrina Khanam, ${ }^{3}$ (D) Jonatan Slotte, ${ }^{3}$ (D) Ilja Makkonen, ${ }^{3}$ (D) Geoffrey Pourtois, ${ }^{2,4}$ Clement Porret, ${ }^{2}$ (D) Roger Loo, ${ }^{2}$ (D) and Wilfried Vandervorst ${ }^{1,2}$

\author{
AFFILIATIONS \\ ${ }^{1}$ Institute for Nuclear and Radiation Physics, K.U. Leuven, Celestijnenlaan 200D, B-3001 Leuven, Belgium \\ ${ }^{2}$ Imec vzw, Kapeldreef 75, B-3001 Leuven, Belgium \\ ${ }^{3}$ Department of Applied Physics, Aalto University, P.O. Box 15100, Fl-00076 Aalto, Finland \\ ${ }^{4}$ Department of Chemistry, Plasmant Research Group, University of Antwerp, B-2610 Wilrijk-Antwerp, Belgium
}

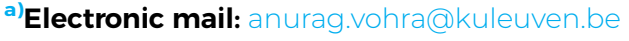

\begin{abstract}
We examined the vacancy trapping proficiency of $\mathrm{Sn}$ and $\mathrm{P}$ atoms in germanium using positron annihilation spectroscopy measurements, sensitive to the open-volume defects. Epitaxial $\mathrm{Ge}_{1-x} \mathrm{Sn}_{x}$ films were grown by chemical vapor deposition with different $\mathrm{P}$ concentrations in the $\sim 3.0 \times 10^{19}-1.5 \times 10^{20} \mathrm{~cm}^{-3}$ range. We corroborate our findings with first principles simulations. Codoping of Ge with a Sn concentration of up to $9 \%$ is not an efficient method to suppress the free vacancy concentration and the formation of larger phosphorus-vacancy complexes. Experimental results confirm an increase in the number of $\mathrm{P}$ atoms around the monovacancy with $\mathrm{P}$-doping, leading to dopant deactivation in epitaxial germanium-tin layers with similar Sn content. Vice versa, no impact on the improvement of maximum achieved $\mathrm{P}$ activation in Ge with increasing Sn-doping has been observed. Theoretical calculations also confirm that $\mathrm{P}_{n}-\mathrm{V}$ (vacancy) complexes are energetically more stable than the corresponding $\mathrm{Sn}_{m} \mathrm{P}_{n}-\mathrm{V}$ and $\mathrm{Sn}_{m}-\mathrm{V}$ defect structures with the same number of alien atoms (Sn or P) around the monovacancy. The strong attraction of vacancies to the phosphorus atoms remains the dominant dopant deactivation mechanism in Ge as well as in $\mathrm{Ge}_{1-x} \mathrm{Sn}_{x}$.
\end{abstract}

Published under license by AIP Publishing. https://doi.org/10.1063/1.5107503

\section{INTRODUCTION}

Vacancies $(\mathrm{V})$ are vehicles for phosphorus $(\mathrm{P})$ diffusion in germanium $(\mathrm{Ge})$ leading to the formation of mobile $\left(\mathrm{P}_{1}-\mathrm{V}\right)^{-1}$ pairs. ${ }^{1,2}$ Their interaction with the positively charged substitutional $\mathrm{P}^{+}$ atoms forms immobile $\mathrm{P}_{n}-\mathrm{V}(n>1)$ clusters leading to a mechanism of dopant deactivation in $\mathrm{Ge}^{3}$ Point defect engineering strategies are hence required to decelerate the $\mathrm{P}$ diffusion in $\mathrm{Ge}^{4}$ For instance, the addition of a large isovalent tin $(\mathrm{Sn})$ atom can have a repercussion on the migration of $\left(\mathrm{P}_{1}-\mathrm{V}\right)^{-1}$ pairs in Ge. Consequently, the codoping of Ge:P with $\mathrm{Sn}$ (as a trap for vacancies) and $\mathrm{Si}$ (for the correct lattice parameter) as a source-drain (S/D) material for $n$-Ge FinFETs might enable the combination of high conductivity with the introduction of preferred tensile strain in the Ge channel to boost carrier mobility.
Tahini et $a l .{ }^{4}$ investigated the impact of oversized codopants, $\mathrm{Sn}$ and $\mathrm{Hf}$, to retard the vacancy-mediated diffusion of $\mathrm{P}$ atoms in Ge. The first principles simulations predicted that the codoping with $\mathrm{Sn}$ increases the migration energy barriers via the formation of strongly bound $\left(\mathrm{Sn}_{1} \mathrm{P}_{1}-\mathrm{V}\right)^{-1}$ clusters, which indeed could trap the migrating $\left(\mathrm{P}_{1}-\mathrm{V}\right)^{-1}$ pairs. ${ }^{4}$ However, the interplay between the $\mathrm{Sn}, \mathrm{P}$ and the vacancy, which is a dominant point defect in $\mathrm{Ge}$, and their contribution toward the dopant activation requires more understanding, both theoretically and experimentally. In order to gain insight into their interaction, we examined the vacancy trapping proficiency of $\mathrm{Sn}$ and $\mathrm{P}$ atoms in Ge using positron annihilation spectroscopy (PAS), which is sensitive to the open-volume defects, on $\mathrm{Ge}_{1-x} \mathrm{Sn}_{x}$ :P films and corroborate our findings with the first principles simulations. 
In this work, we determined the role of vacancies in the formation of $\mathrm{P}_{n}-\mathrm{V}, \mathrm{Sn}_{m}-\mathrm{V}$, and $\mathrm{Sn}_{m} \mathrm{P}_{n}-\mathrm{V}$ clusters and the impact of Sn-doping on the doping activation in Ge. Our findings show that the concentration of monovacancy defects in as-grown $\mathrm{Ge}_{1-x} \mathrm{Sn}_{x}$ epitaxial layers increases with $\mathrm{P}$-doping. In the presence of vacancies, the dopants can cluster with them to form E-centers. ${ }^{3}$ For $\mathrm{P}$ concentrations above $8 \times 10^{19} \mathrm{~cm}^{-3}$ in as-grown $\mathrm{Ge}_{1-x} \mathrm{Sn}_{x}$ layers, the monovacancies are the dominant open-volume defect decorated with at least $3 \mathrm{P}$ atoms. PAS measurements give clear evidence for an increase in the number of $\mathrm{P}$ atoms as the first nearest neighbor $(1 \mathrm{nn})$ to the vacancy with increased P-doping. Furthermore, PAS results corroborating with the first principles simulations suggest that there is at least $1 \mathrm{Sn}$ atom present around the monovacancy. However, for the higher $\mathrm{P}$ concentrations $\left(>1 \times 10^{20} \mathrm{~cm}^{-3}\right)$ in $\mathrm{Ge}_{1-x} \mathrm{Sn}_{x}$ layers, the measured annihilation spectrum is dominated mainly by the $\mathrm{P}$ atoms present around the monovacancy. Based on these observations, we conclude that the codoping of Ge with $\mathrm{Sn}$ has a negligible effect on the improvement of $\mathrm{P}$ activation in $\mathrm{Ge}$ for Sn concentrations up to $9 \%$, in essence that vacancies have more attraction for $\mathrm{P}$ atoms as compared to $\mathrm{Sn}$ atoms. The strong interaction of phosphorus with vacancies is still the major contributor toward the dopant deactivation in $\mathrm{Ge}$ as well as in $\mathrm{Ge}_{1-x} \mathrm{Sn}_{x}$.

\section{COMPUTATIONAL DETAILS}

The first principles simulations were carried out using planewave density functional theory (DFT) as implemented in the Vienna $a b$ initio Simulation Package (VASP). ${ }^{7,8}$ The core electrons were treated using pseudopotentials generated according to the projector augmented wave (PAW) method with a kinetic energy cutoff of $300 \mathrm{eV}$ and using a cubic $216 \mathrm{Ge}$ atoms supercell with sampling of the Brillouin zone (BZ) at the $\Gamma$ point. ${ }^{9,10}$ A Heyd-ScuseriaErnzerhof (HSE06) ${ }^{11}$ hybrid functional was used with a default fraction of its nonlocal Hartree-Fock potential (25\%) to match the experimental value of the indirect bandgap of $\mathrm{Ge}(0.74 \mathrm{eV})$ at $0 \mathrm{~K}$. The convergence criteria for self-consistency of the electronic cycles and forces on each atom at the end of the simulation were $1 \times 10^{-6} \mathrm{eV}$ and $1 \times 10^{-3} \mathrm{eV} / \AA$, respectively.

The annihilation characteristics for positrons trapped inside the open-volume defects such as $\mathrm{P}_{n}-\mathrm{V}, \mathrm{Sn}_{m}-\mathrm{V}, \mathrm{Sn}_{m}-\mathrm{V}_{2}$, and $\mathrm{Sn}_{m} \mathrm{P}_{n}-\mathrm{V}$ clusters were modeled using the so-called "conventional-scheme" ${ }^{\text {"12 }}$ $(\mathrm{CS})$ within the zero-positron-density $\left(n_{+} \rightarrow 0\right)$ limit of the twocomponent density functional theory. ${ }^{13}$ The repulsive forces of positrons on the ions were taken into consideration while relaxing the defect structures. ${ }^{14}$ We chose a state-dependent scheme for calculating the momentum distributions of the annihilating electronpositron pairs. ${ }^{15}$ The annihilation rates were calculated within the state-dependent scheme using the local-density approximation enhancement factor parameterized by Boroński and Nieminen. ${ }^{13}$ We would highly recommend the reader to read our previous publications (Refs. 14 and 16) for elaborative explanations about our computational technique on the modeling of annihilating electron-positron pairs in solids.

\section{STABILITY OF $\mathbf{P}_{n}-\mathbf{V}, \mathbf{S n}_{m}-\mathrm{V}$, and $\mathrm{Sn}_{m} \mathbf{P}_{n}-\mathrm{V}$ CLUSTERS}

Tahini et al. ${ }^{4}$ recently reported that the $\left(\mathrm{Sn}_{1} \mathrm{P}_{1}-\mathrm{V}\right)^{-1}$ cluster is more stable than the $\left(\mathrm{P}_{1}-\mathrm{V}\right)^{-1}$ pair because of its stronger binding energy $\left(E_{b}\right)$ value and could retard the further vacancy-mediated diffusion of the latter. The presence of $\mathrm{Sn}$ passivation around the vacancy indeed leads to an increased migration energy of the $\left(\mathrm{Sn}_{1} \mathrm{P}_{1}-\mathrm{V}\right)^{-1}$ pair as compared to the $\left(\mathrm{P}_{1}-\mathrm{V}\right)^{-1}$ pair. ${ }^{3,4}$ However, the analysis in Ref. 4 is based on only single $\mathrm{P}$ and $\mathrm{Sn}$ atoms around the vacancy. The relative stabilities of $\mathrm{P}_{n}-\mathrm{V}, \mathrm{Sn}_{m}-\mathrm{V}$, and the mixed $\mathrm{Sn}_{m} \mathrm{P}_{n}-\mathrm{V}$ clusters with more than one $\mathrm{Sn}$ or $\mathrm{P}$ atoms around the monovacancy have not been reported earlier. To gain insights into the most representative atomic configurations from an energetic perspective, we systematically relaxed the atomic structures of all possible atomic conformations accounting for vacancy, $\mathrm{P}$, and $\mathrm{Sn}$ atoms, given that both $\mathrm{Sn}$ and $\mathrm{P}$ atoms have affinity toward vacancies. In this case, the energy of the different configurations is the lowest when either the $\mathrm{Sn}$ or the $\mathrm{P}$ atom is placed as a $1 \mathrm{nn}$ with respect to the position of the vacancy. The attraction between the codopants and the vacancy is evaluated by calculating their binding energies using Eq. (2) in Ref. 4. A negative binding energy value indicates that a defect cluster is stable with respect to its constituent point defects. ${ }^{4}$

The calculated binding energies of $\mathrm{P}_{n}-\mathrm{V},{ }^{17} \mathrm{Sn}_{m}-\mathrm{V}$, and mixed $\mathrm{Sn}_{m} \mathrm{P}_{n}-\mathrm{V}$ clusters are shown in Fig. 1. The negative binding energy values exhibited by $\mathrm{Sn}_{m}-\mathrm{V}$ complexes predict that the $\mathrm{Sn}$ atoms are bound to the vacancies. However, the $E_{b}$ values for atomic arrangements involving either only $\mathrm{P}$ atoms or both $\mathrm{Sn}$ and $\mathrm{P}$ atoms around the monovacancy indicate that they are energetically more stable than the analogous configurations with $\mathrm{Sn}$ atoms alone. As a consequence, the mixed $\operatorname{Sn}_{m} \mathrm{P}_{n}-\mathrm{V}$ clusters will have tendency to be P-rich, since they are energetically more favorable to occur as more phosphorus is incorporated in as-grown epitaxial $\mathrm{Ge}_{1-x} \mathrm{Sn}_{x}$ layers.

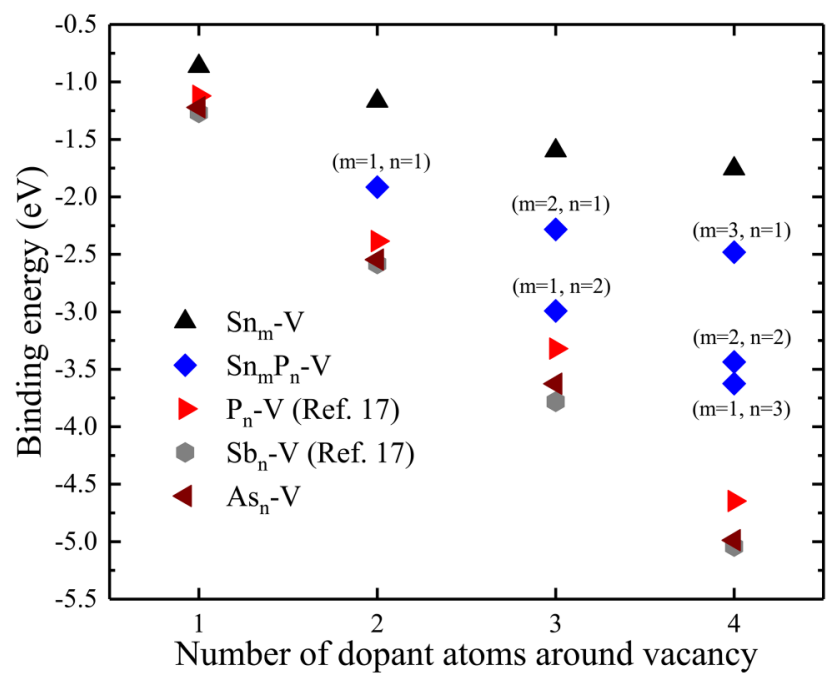

FIG. 1. Calculated binding energies $\left(E_{b}\right)$ of $P_{n}-V,{ }^{17} S n_{m}-V$, and $S n_{m} P_{n}-V$ clusters for the neutral vacancy with respect to its isolated constituents in Ge. $E_{b}$ values for alternative dopant species ( $\mathrm{Sb}$ and $\mathrm{As}$ ) as $1 \mathrm{nn}$ to monovacancy are also shown. 
TABLE I. Layer properties: thickness $(t)$, Sn content, Hall carrier concentration, and the total $\mathrm{P}$ concentration for as-grown GeSn:P films.

\begin{tabular}{lcccc}
\hline \hline Sample & $\begin{array}{c}t_{\text {SIMS }} \\
(\mathrm{nm})\end{array}$ & $\begin{array}{c}\mathrm{Sn}_{\text {RBS }} \\
(\text { at. \%) }\end{array}$ & $\begin{array}{c}\text { Hall conc. } \\
\left(\mathrm{cm}^{-3}\right)\end{array}$ & $\begin{array}{c}\text { P total } \\
\left(\mathrm{cm}^{-3}\right)\end{array}$ \\
\hline $\mathrm{A}^{\mathrm{a}}$ & 81 & 6.4 & $3.6 \times 10^{19}$ & $3.4 \times 10^{19}$ \\
$\mathrm{~B}$ & 77 & 7.0 & $6.6 \times 10^{19}$ & $8.1 \times 10^{19}$ \\
$\mathrm{C}$ & 78 & 6.6 & $4.7 \times 10^{19}$ & $1.5 \times 10^{20}$ \\
$\mathrm{Ge}(\mathrm{P})^{\mathrm{b}}$ & 130 & 0 & $5.7 \times 10^{19}$ & $1.4 \times 10^{20}$ \\
\hline
\end{tabular}

${ }^{a}$ The slight deviation in the active carrier concentration from the total $\mathrm{P}$ content in sample A is most likely due to the underestimation of the Hall scattering factor.

${ }^{\mathrm{b}} \mathrm{Ge}: \mathrm{P}$ sample was grown using $\mathrm{Ge}_{2} \mathrm{H}_{6}$ at $320^{\circ} \mathrm{C} .{ }^{19}$

\section{EXPERIMENTAL DETAILS}

The results obtained based on our first principles simulations for the atomic interactions between Sn, P, and vacancy within the Ge lattice have been benchmarked against PAS measurements on $\mathrm{Ge}_{1-x} \mathrm{Sn}_{x}: \mathrm{P}$ epitaxial films. The layers were epitaxially grown at $315^{\circ} \mathrm{C}$ on top of $600 \mathrm{~nm}$ thick Ge-buffered $\mathrm{Si}$ (001) substrates using $\mathrm{GeH}_{4}, \mathrm{SnCl}_{4}$, and $\mathrm{PH}_{3}$ as gas precursors. ${ }^{18}$ For a fixed $\mathrm{SnCl}_{4}$ flow, the partial pressure of $\mathrm{PH}_{3}$ was tuned to reach different $\mathrm{P}$ concentration levels in $\mathrm{Ge}_{1-x} \mathrm{Sn}_{x}$ layers. However, the maximum $\mathrm{P}$ activation in $\mathrm{Ge}_{1-x} \mathrm{Sn}_{x}$ layers is limited to $\sim 6 \times 10^{19} \mathrm{~cm}^{-3}$ and saturates around the similar values independent of the Sn concentration as studied for $\mathrm{Sn}$ contents up to $9 \%$. Hence, we restrict our discussions on PAS measurements to $\mathrm{Ge}_{1-x} \mathrm{Sn}_{x}$ layers with a $\mathrm{Sn}$ concentration of $6.4-7 \%$ (from here on referred as "GeSn:P"). Table I shows the Sn percentage as measured by Rutherford Backscattering Spectroscopy (RBS), the active carrier concentrations as extracted from $\mu$ Hall measurements and the total P concentration and the film thicknesses from secondary ion mass spectrometry (SIMS). A Hall scattering factor (HSF) of 1 has been assumed in this study.

To study the dominant open-volume defects and their chemical environment in the GeSn:P films, the Doppler Broadening (DOBS) and the Coincidence Doppler Broadening Spectroscopy $(\mathrm{CDOBS})^{20}$ measurements have been used. The shapes of the annihilation spectra were characterized by using the conventional line shape parameters $S$ and $W$, with integration windows fixed at $(0<|p|<0.5$ a.u.) for $S$ parameter and $(1.5<|p|<3.5$ a.u. $)$ for $W$ parameter, where $p$ stands for the Doppler shift in terms of momentum and a.u. for atomic units. All samples in Table I were measured with CDOBS setup, using positron implantation energy of $4.5 \mathrm{keV}$. A positron-trap-free germanium sample was used as a reference to normalize the CDOBS data. For a detailed description of the PAS measurement technique, the reader is referred to Refs. 12,21, and 22.

\section{DOPPLER BROADENING MEASUREMENTS}

The $W$ parameters as a function of the positron implantation energy for different P-doped GeSn layers are shown in Fig. 2(a). A P-doped Ge sample [from here on referred as " $\mathrm{Ge}(\mathrm{P})$ "] grown on top of the same Ge template as GeSn:P layers is also shown to differentiate the effect of Sn-doping on positron trapping. At $E \sim 0.5 \mathrm{keV}$, the $W$ parameters are most sensitive to the surface annihilation. At $E=3.5-5 \mathrm{keV}$, the $W$ parameter describes positron annihilation mainly in the GeSn:P layers. The measured $W$ parameters for the energy value around $10 \mathrm{keV}$, correspond mostly to the annihilation of positrons in the underlying Ge-virtual
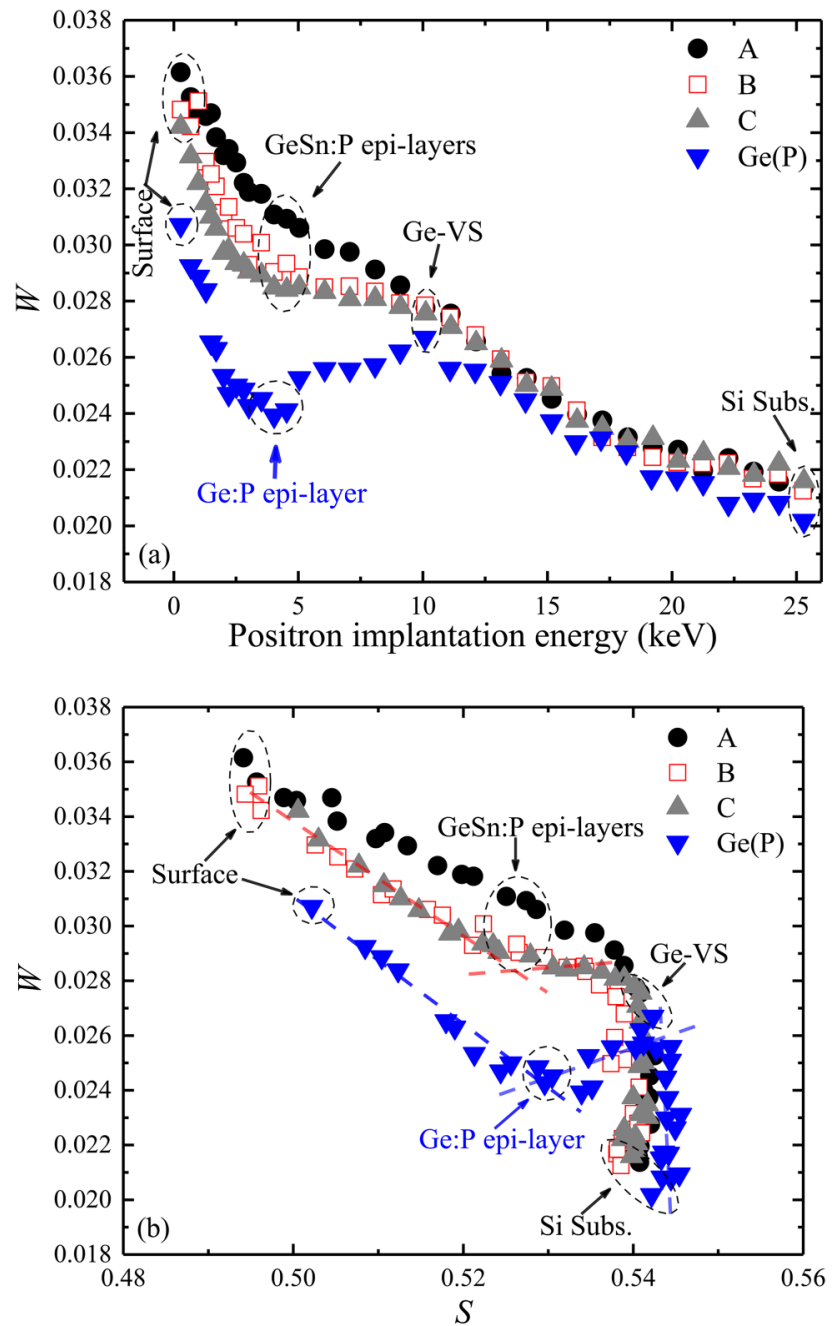

FIG. 2. (a) $W$ parameters vs positron implantation energy and (b) $W(S)$-plot for $\mathrm{GeSn}$ samples with different $P$ concentrations (Table I). A Ge(P) layer with a similar $\mathrm{P}$ concentration as of sample $\mathrm{C}$ is included to differentiate the effect of $\mathrm{Sn}$-doping on positron trapping. The annihilation states at the surface, in the $\mathrm{Ge:}$ $P$ layer, in the GeSn:P layers, in the Ge-VS, and in the Si substrate are marked by arrows. The Ge:P layer with the similar active doping and the total $P$ concentration as in the GeSn:P layer (i.e., sample $C$ ) shows distinguished and strong positron trapping as compared to weakly defined annihilation state in the GeSn layer. The error bars for the measured $S$ and $W$ parameters are \pm 0.0005 and 0.0001 , respectively. 
substrate (VS). The decrease in $W$ values above $10 \mathrm{keV}$ is due to positrons annihilating in the silicon substrate.

The measured $W$ parameters in Fig. 2(a) exhibit a steep slope throughout the as-grown GeSn:P epilayers (i.e., 0.5-5.0 keV). This is due to the narrow implantation profile of positrons at low energies, ${ }^{23,24}$ combined with the short positron diffusion lengths. The effective positron diffusion lengths are considerably smaller for all samples considered in this study as compared to the defect-free Ge-reference. This indeed signifies that the concentration of openvolume defects is much higher (above $5 \times 10^{18} \mathrm{~cm}^{-3}$ ) than the sensitivity limit of PAS measurements causing saturation like trapping (i.e., effectively all positrons annihilate at the open-volume defects) in as-grown GeSn:P epilayers. This in point of fact also means that for the $\mathrm{P}$-doping ranges considered in this study (above $1 \times 10^{19} \mathrm{~cm}^{-3}$ ), the $W$ parameter is only sensitive to the changes in the chemical environment of the dominating open-volume defects rather than to their total concentration.

However, despite saturation like trapping, an evident dependence has been observed for P-doping in GeSn layers. The increase in the steepness of the $W$ parameter slope from the surface state to the annihilation state in the GeSn epilayers indicates a decrease in effective positron diffusion lengths with increased P-doping. This decrease in positron diffusion lengths corresponds to an increase in the concentration of open-volume defects in the GeSn:P layers. Similar trends were observed for the P-doped Ge layers, where the concentration of monovacancy defects also increases with P-doping. ${ }^{17}$ Since, monovacancies alone are unstable in $\mathrm{Ge}$ above $200 \mathrm{~K},{ }^{25}$ the majority of vacancies are decorated by $\mathrm{P}$ atoms. Our recent study on the evolution of vacancydonor complexes in heavily P-doped epitaxial Ge films confirms that for a total $\mathrm{P}$ concentration in the $6 \times 10^{19}-1 \times 10^{20} \mathrm{~cm}^{-3}$ range, there are at least 2-3 $\mathrm{P}$ atoms around each monovacancy sized open-volume defect. ${ }^{17}$ The occurrence of $\mathrm{P}_{n}-\mathrm{V}$ clusters is the dominant dopant deactivation mechanism in Ge.

Furthermore, the annihilation states as indicated by arrows in GeSn:P layers are not as pronounced as in the Ge:P layer [Fig. 2(a)]. By closely looking at the $W$ parameter values as measured for the highly doped $\mathrm{Ge}(\mathrm{P})$ sample with a similar $\mathrm{P}$ concentration as in GeSn:P (i.e., sample C), a clear slope is visible from the annihilation state in the Ge:P epilayer toward the annihilation state in the Ge-VS. This effect is better visible from the line shape parameters in the $S-W$ plane as shown in Fig. 2(b). For the $\mathrm{Ge}(\mathrm{P})$ sample, the measurement data in Fig. 2(b) lie on three separate segments as indicated by the dotted lines. The first segment corresponds to the superposition between the surface state and the trap state in the Ge:P layer. The second segment corresponds to the superposition between the trap state in the Ge:P layer and the Ge-VS. The third segment belongs to the superposition between the trap state in Ge-VS and the $\mathrm{Si}$ substrate. The annihilation states in P-doped epitaxial Ge layers are clearly distinguishable for P-doping levels above $6 \times 10^{19} \mathrm{~cm}^{-3}$, ${ }^{17}$ whereas for GeSn layers, the annihilation state in the $S$ - $W$ plane is barely visible for sample A. Although, from the $W-E$ plot a kink at $\sim 4 \mathrm{keV}$ gives a hint for the presence of annihilation state in sample A. For samples B and $\mathrm{C}$, there exists a clear change in the slopes between the surface to the epilayer trap states and the epilayer to the Ge-VS trap states. However, they are not as clearly defined as in the $\mathrm{Ge}(\mathrm{P})$ sample. This observation indicates that the positron trapping is either relatively weaker in GeSn:P layers as compared to the P-doped Ge films or that the annihilation state is different due to the presence of Sn either at $1 \mathrm{nn}$ sites around the open-volume defects or at the substitutional lattice sites in the immediate vicinity of the defects.

\section{POSITRON TRAPPING IN $\mathrm{P}_{n}-\mathrm{V}, \mathrm{Sn}_{m}-\mathrm{V}$, and $\mathrm{Sn}_{m} \mathrm{P}_{n}-\mathrm{V}$ CLUSTERS}

To understand the DOBS data in Fig. 2, we modeled the trapping of positrons inside the monovacancy or divacancy defects decorated by $\mathrm{Sn}$ and $\mathrm{P}$ atoms as $\mathrm{Inn}$ (i.e., for the minimum energy configurations reported in Fig. 1). Calculated positron densities (gray, glassy isosurface) in Fig. 3 illustrate the positron trapping into $\mathrm{P}_{1}-\mathrm{V}$ (P atom in green), $\mathrm{Sn}_{1}-\mathrm{V}$ (Sn atom in blue) with the $\mathrm{Sn}$ atom in the split-vacancy configuration, $S_{1} \mathrm{P}_{1}-\mathrm{V}, \mathrm{Sn}_{1} \mathrm{P}_{2}-\mathrm{V}, \mathrm{Sn}_{1} \mathrm{P}_{3}-\mathrm{V}$, and
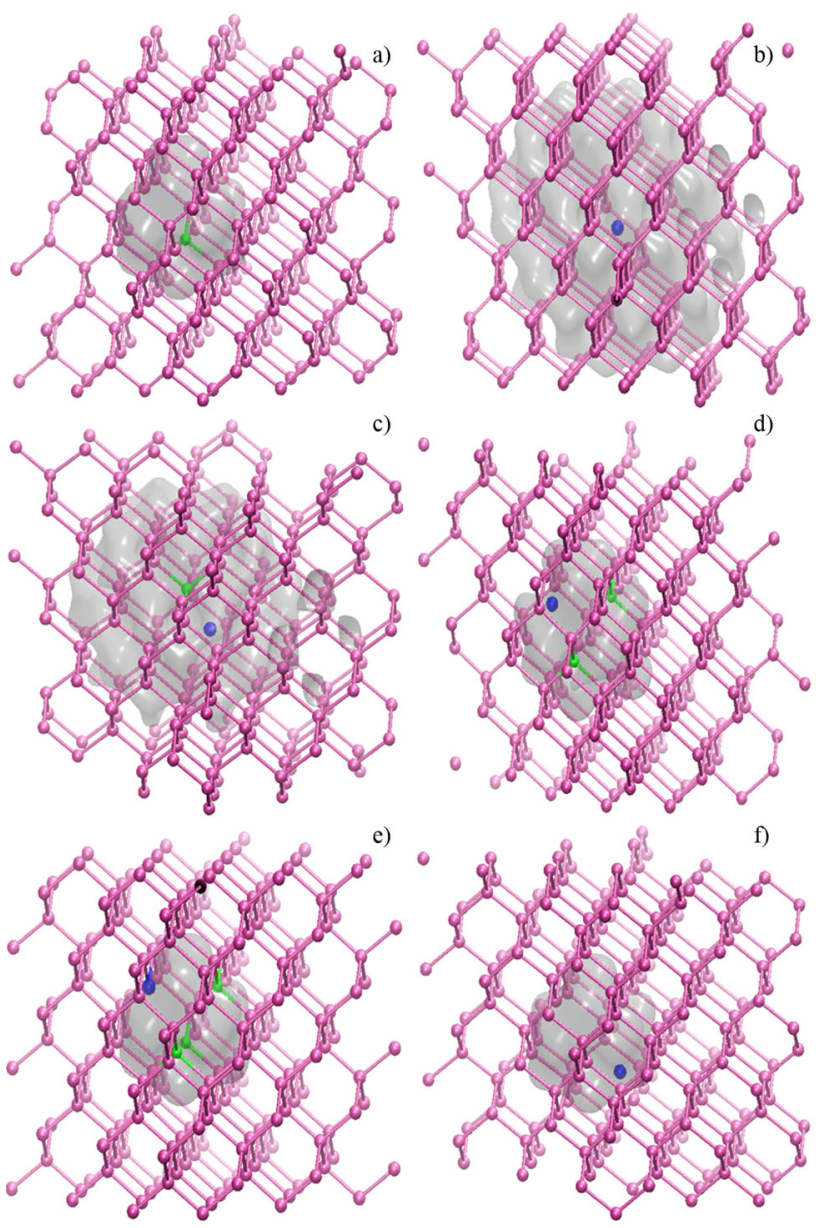

FIG. 3. Calculated positron densities (gray, glassy isosurface with an isovalue of $2 \times 10^{-3} \AA^{-3}$ ) for relaxed $P_{1}-V$ (P atom in green), $S n_{1}-V$ (Sn atom in blue), $S n_{1} P_{1}-V, S n_{1} P_{2}-V, S n_{1} P_{3}-V$, and $S n_{1}-V_{2}$ configurations in the Ge lattice. The positron density is localized within the open volume for $P_{1}-V, S n_{1} P_{2}-V, S n_{1} P_{3}-V$, and $S n_{1}-V_{2}$ clusters [(a), (d), (e), and (f)], whereas it is delocalized for $S n_{1}-V$ and $\mathrm{Sn}_{1} \mathrm{P}_{1}-\mathrm{V}$ complexes $[(\mathrm{b})$ and $(\mathrm{c})]$. 
$\mathrm{Sn}_{1}-\mathrm{V}_{2}$ defect structures. Typically, positrons are localized at $\mathrm{P}_{n}-\mathrm{V}$ defects [as shown in Fig. 3(a) for $\mathrm{P}_{1}-\mathrm{V}$ complex], whereas no positron localization occurs for $\mathrm{Sn}_{m}-\mathrm{V}$ clusters [as shown in Fig. 3(b) for $\mathrm{Sn}_{1}-\mathrm{V}$ complex]. In the case of $\mathrm{Sn}_{m} \mathrm{P}_{n}-\mathrm{V}$ complexes [Figs. 3(c)-3(e)], the positron remains localized within the open volume only for the defect structures with at least $2 \mathrm{P}$ atoms around the monovacancy. However, for a larger open-volume defect, e.g., a divacancy $\left(V_{2}\right)$, positron is localized within the open volume as shown in Fig. 3(f) for the $\mathrm{Sn}_{1}-\mathrm{V}_{2}$ defect structure.

The relaxation of $1 \mathrm{nn}$ atoms around the monovacancy plays an important role for positron trapping in $\mathrm{P}_{n}-\mathrm{V}, \mathrm{Sn}_{m}-\mathrm{V}$, and $\mathrm{Sn}_{m} \mathrm{P}_{n}-\mathrm{V}$ complexes. In the case of $\mathrm{P}_{n}-\mathrm{V}$ clusters, the $\mathrm{P}$ atoms relax in the outward direction with respect to the vacancy, which increases the open-volume of the defect. This indeed means that as the number of $\mathrm{P}$ atoms increases around the monovacancy, the positron annihilation state becomes more pronounced in experimentally measured annihilation spectra for heavily P-doped Ge films. ${ }^{17}$ On the other hand for $\mathrm{Sn}_{m}-\mathrm{V}$ clusters, the atoms around the monovacancy relax inward, which reduces the open volume of the defect. In this case, the size of the open volume becomes considerably small in order to trap the positron, whereas in the case of $\mathrm{Sn}_{m} \mathrm{P}_{n}$-V complexes, atoms around the monovacancy follow both inward ( $\mathrm{Sn}$ atoms) and outward ( $\mathrm{P}$ atoms) relaxation. In this scenario, the positron remains trapped within the open volume of the defect if there are at least $2 \mathrm{P}$ atoms present around the monovacancy.

As already discussed in Sec. V, positrons are in saturation trapping with the dominating open-volume defects in both Ge:P and GeSn:P layers. The upward shift in the annihilation state of the GeSn:P layer (i.e., sample C) with similar total P concentration and fairly the same concentration of the open-volume defects (due to the identical steepness of the $W$ parameter slope) as in the $\mathrm{Ge}(\mathrm{P})$ sample indeed signifies that the chemical environment of the dominating open-volume defect is different in both the layers (Fig. 2). The simulation results predicting positron trapping in Sn-related defects (e.g., $\mathrm{Sn}_{1} \mathrm{P}_{2}-\mathrm{V}$ ) with a reduced size of the open volume will result in higher $W$ parameters for GeSn:P layers as compared to positron trapping in $\mathrm{P}_{n}-\mathrm{V}$ clusters with relatively larger size of the open volume in Ge:P layers. This result indeed also gives the first indication for the presence of $\mathrm{Sn}$ atoms around the dominating open-volume defect in GeSn:P layers.

Furthermore, the annihilation states for $\mathrm{P}_{n}-\mathrm{V}$ clusters itself can differ in $\mathrm{Ge}_{1-x} \mathrm{Sn}_{x}$ layers by relaxation effects induced due to the presence of $\mathrm{Sn}$ atoms at the nearest neighbor sites $(\geq 2 \mathrm{nn}$ from the monovacancy). Our simulations suggest that the positrons are localized at Sn-related defects with divacancies as the size of the open-volume defect is large enough to trap them. However, the experimental results from CDOBS measurements as discussed below, confirm that the dominating open-volume defects in as-grown epitaxial GeSn:P layers are not bigger than the size of a monovacancy. Hence, divacancy related defects can be ruled out as the positron traps in as-grown $\mathrm{Ge}(\mathrm{P})$ and $\mathrm{GeSn}: \mathrm{P}$ samples.

\section{COINCIDENCE DOPPLER BROADENING MEASUREMENTS}

Additional CDOBS measurements allow to determine the dominant defect type and its chemical environment in as-grown
GeSn:P epitaxial layers. An incident positron energy of $4.5 \mathrm{keV}$ was used to measure all the samples. At $4.5 \mathrm{keV}$, the annihilation spectrum corresponds mainly to the positrons annihilating within the GeSn:P layers and the $\mathrm{Ge}(\mathrm{P})$ sample [Fig. 2(a)]. Figure 4 shows annihilation intensities as a function of momentum in the form of ratio curves (normalized to a defect-free Ge-reference sample) for $\mathrm{GeSn}$ layers with different $\mathrm{P}$ concentrations. The intensities measured in the peak region $(1<p<1.5$ a.u.) confirm the presence of monovacancy defects for in situ P-doped Ge as well as GeSn layers. Typically, lower intensities are measured in the peak region for the larger open-volume defects, $\mathrm{V}_{x}(x \geq 2)$, as demonstrated both experimentally and using first principles simulations for $\mathrm{As}-\mathrm{V}_{2}$ complexes in As-implanted and laser annealed $\mathrm{Ge}^{26}$

Furthermore, the decrease in the intensity with P-doping for GeSn layers at high momenta ( $p>1.5$ a.u.) substantiates that the number of $\mathrm{P}$ atoms around the monovacancy increases with the increasing P-doping. This decrease in the intensity at high momenta is due to a decreased annihilation probability of positrons with the $3 d$ electron shell of Ge. The replacement of Ge atoms around the monovacancy by $\mathrm{P}$ atoms leads to a further omission of the $3 d$ electron shell as the phosphorus atoms consist of only $s$ and $p$ orbitals. The dominating monovacancy defects in as-grown GeSn: $\mathrm{P}$ layers are passivated by at least 2 (in sample A) and at least 3 (in samples $\mathrm{B}$ and $\mathrm{C}$ ) $\mathrm{P}$ atoms. These results are in line with our recent PAS studies on epitaxial Ge:P films. ${ }^{17}$ For detailed explanations on

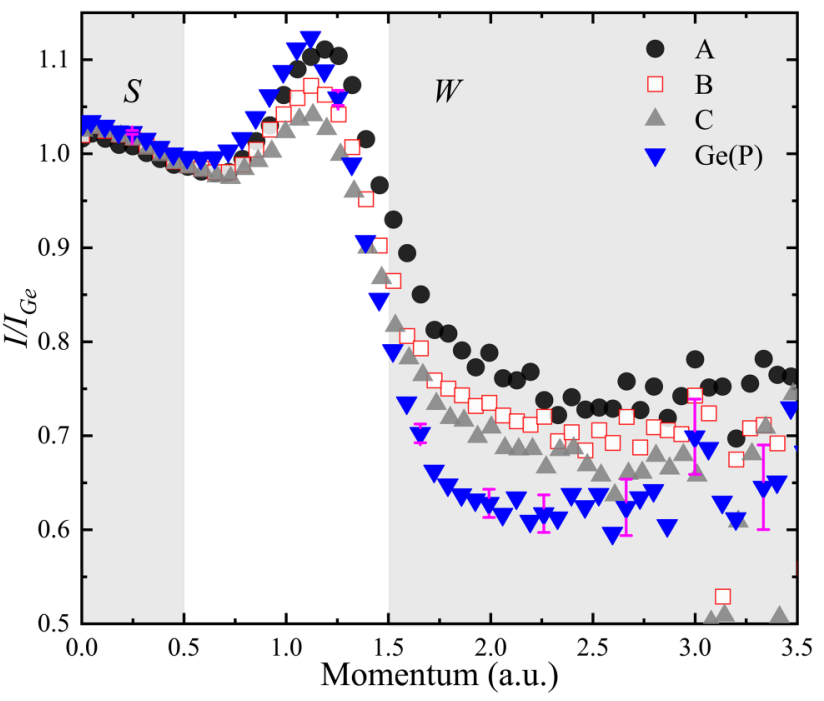

FIG. 4. Annihilation intensities (normalized to a defect-free Ge-reference) for GeSn:P epitaxial layers with different $P$ concentrations, measured at an incident positron energy of $4.5 \mathrm{keV}$. The integration windows for $S$ and $W$ parameters are indicated by the shaded regions. To distinguish the effect of Sn-doping, the ratio plot for the $\mathrm{Ge}(\mathrm{P})$ sample has been included, where the dominant openvolume defects are monovacancies passivated with at least $3 \mathrm{P}$ atoms. Error bars for $\mathrm{Ge}(\mathrm{P})$ sample show the uncertainty in the measured spectrum increases due to lesser number of counts at higher momenta ( $>2.5$ a.u., where a.u. stands for atomic units). 
the experimental evidence for the evolution of phosphorusvacancy clusters in epitaxial Ge, the reader is referred to Ref. 17.

It must be noted that the intensity at high momenta is clearly the lowest for the $\mathrm{Ge}(\mathrm{P})$ sample with similar $\mathrm{P}$ concentration as in the $\mathrm{GeSn}$ layer (i.e., sample $\mathrm{C}$ ). Although, there are at least $3 \mathrm{P}$ atoms present around the monovacancies in both samples (Fig. 4), still sample $C$ exhibits a higher intensity at high momenta in comparison to the $\mathrm{Ge}(\mathrm{P})$ sample. This observation indeed supports our previous argument in Sec. VI that the presence of Sn atoms around the monovacancy or at the substitutional lattice sites $(\geq 2 \mathrm{nn}$ from the monovacancy) affects the annihilation state in GeSn:P layers (Fig. 2). In order to interpret the CDOBS spectra, we calculated the ratio plots for $\mathrm{Sn}_{m} \mathrm{P}_{n}-\mathrm{V}$ complexes where positron remains trapped within the open volume of the defect structure as shown in Fig. 5. For comparison with $\mathrm{Sn}_{m} \mathrm{P}_{n}-\mathrm{V}$ complexes, the calculated annihilation spectra for $\mathrm{P}_{2}-\mathrm{V}$ and $\mathrm{P}_{3}-\mathrm{V}$ clusters in a bulk Ge lattice are also shown from Ref. 17.

The increased intensity at high momenta for the GeSn layer (i.e., sample $\mathrm{C}$ ) with the similar $\mathrm{P}$ concentration as in $\mathrm{Ge}(\mathrm{P})$ sample can be explained due to several factors. The dominating open-volume defect in both layers is a monovacancy passivated with at least $3 \mathrm{P}$ atoms. In the case of Sn-doping, the presence of single $\mathrm{Sn}$ atom around the monovacancy in a $\mathrm{Sn}_{1} \mathrm{P}_{3}-\mathrm{V}$ cluster instead of the $\mathrm{Ge}$ atom in a $\mathrm{P}_{3}-\mathrm{V}$ complex slightly increases the intensity at high momenta. This increase in the intensity is mainly attributed to the further inward relaxation of the $\mathrm{Sn}$ atom as compared to the Ge atom. However, for $\mathrm{Sn}_{1} \mathrm{P}_{2}-\mathrm{V}$ and $\mathrm{Sn}_{2} \mathrm{P}_{2}-\mathrm{V}$ complexes, certainly the increase in the intensity at high momenta is substantial in comparison to the $\mathrm{Sn}_{1} \mathrm{P}_{3}-\mathrm{V}$ cluster. The experimental spectrum shown in Fig. 4 for sample $C$ is a superposition of

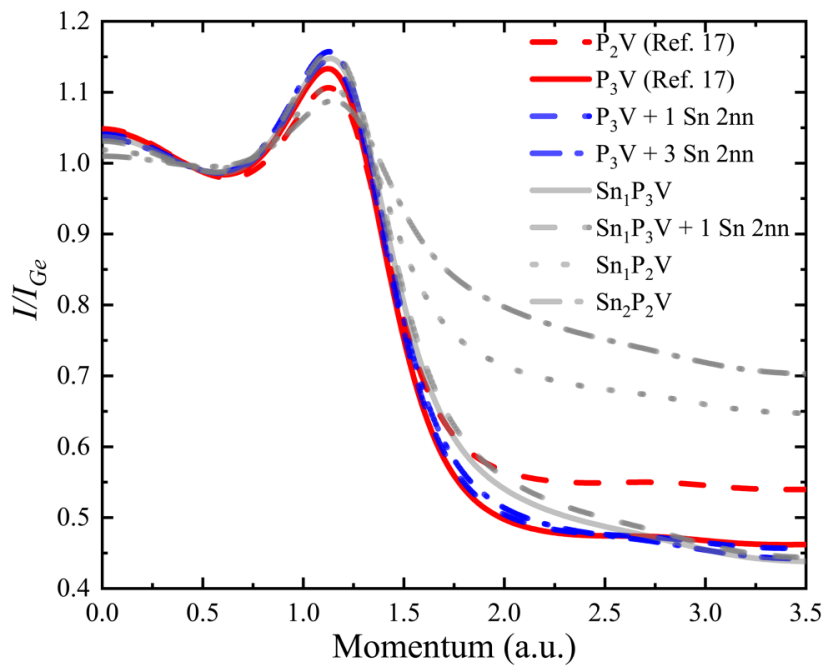

FIG. 5. Calculated ratio plots (scaled to defect-free $216 \mathrm{Ge}$ atoms supercell) for different $P_{n}-V$ and $S_{m} P_{n}-V$ complexes where positron remains trapped within the open volume of the defect structure. The theoretical curves were convoluted with the detector resolution of the experimental CDOBS setup. Momentum values are expressed in atomic units (a.u.). positrons annihilating in $\mathrm{P}_{n}-\mathrm{V}$ and $\mathrm{Sn}_{m} \mathrm{P}_{n}-\mathrm{V}$ clusters. Therefore, the increased intensity at high momenta indicates that there is at least $1 \mathrm{Sn}$ atom present around the monovacancy in GeSn layers. For lower P concentrations, such as in sample A where the dominating defect contains at least $2 \mathrm{P}$ atoms around the monovacancy, there could be 1-2 Sn atoms present as well along with $\mathrm{P}$ atoms. The presence of $\mathrm{Sn}$ atoms at the $2 \mathrm{nn}$ sites with respect to the vacancy (and as $1 \mathrm{nn}$ to the existing $\mathrm{P}$ or $\mathrm{Sn}$ atoms) around the $\mathrm{P}_{n}-\mathrm{V}$ or $\mathrm{Sn}_{m} \mathrm{P}_{n}-\mathrm{V}$ clusters has a marginal effect on the annihilation characteristics at high momenta as shown in Fig. 5.

\section{CONCLUSIONS}

In summary, the strong attraction of monovacancies toward the phosphorus atoms remains the dominant dopant deactivation mechanism in both P-doped $\mathrm{Ge}$ and $\mathrm{Ge}_{1-x} \mathrm{Sn}_{x}$ alloys which has been studied for Sn contents up to $9 \%$. The highest achieved active carrier concentration for $\mathrm{P}$-doped $\mathrm{Ge}_{1-x} \mathrm{Sn}_{x}$ layers is limited to $\sim 6 \times 10^{19} \mathrm{~cm}^{-3}$. Similar active carrier concentrations have been reported for P-doped Ge layers ${ }^{19}$ which were grown with a higher order $\mathrm{Ge}$-precursor (i.e., $\mathrm{Ge}_{2} \mathrm{H}_{6}$ ) and nearly the same growth temperature. Hence, the codoping of Ge:P with a Sn concentration of up to $9 \%$ is not an efficient method to suppress the free vacancy concentration and the formation of larger phosphorus-vacancy complexes.

CDOBS measurements confirm the increase in the number of $\mathrm{P}$ atoms around the monovacancies with increasing $\mathrm{P}$-doping in GeSn layers. These results are consistent with our theoretical predictions as $\mathrm{P}_{n}-\mathrm{V}$ clusters are energetically the most stable ones in Ge (Fig. 1). The energetic favorability for the occurrence of $\mathrm{Sn}_{m} \mathrm{P}_{n}-\mathrm{V}$ complexes is not far behind, when compared to the corresponding $\mathrm{P}_{n}-\mathrm{V}$ clusters. However, any $\mathrm{P}$ atom present in form of $\mathrm{Sn}_{m} \mathrm{P}_{n}-\mathrm{V}$ complexes still remains deactivated in the Ge lattice. In addition, there is a high probability for the $\mathrm{Sn}_{m} \mathrm{P}_{n}-\mathrm{V}$ clusters to be $\mathrm{P}$-rich with increasing P-doping in $\mathrm{Ge}_{1-x} \mathrm{Sn}_{x}$ layers. The CDOBS measurement results for similar P concentration in GeSn (sample C) as in Ge layers provide evidence that there is at least $1 \mathrm{Sn}$ atom present around the monovacancy. However, no remarkable effect on doping activation in $\mathrm{Ge}_{1-x} \mathrm{Sn}_{x}$ layers has been observed due to the presence of single $\mathrm{Sn}$ atom around the vacancies.

The concentration of $\mathrm{P}_{n}-\mathrm{V}, \mathrm{Sn}_{m}-\mathrm{V}$, and $\mathrm{Sn}_{m} \mathrm{P}_{n}-\mathrm{V}$ complexes certainly depends on the total $\mathrm{P}, \mathrm{Sn}$, and vacancy concentrations in the as-grown epitaxial $\mathrm{Ge}_{1-x} \mathrm{Sn}_{x}$ layers. A higher $\mathrm{P}$ activation might be possible for $\mathrm{Ge}_{1-x} \mathrm{Sn}_{x}$ layers with higher $\mathrm{Sn}$ content (above 9\%), as there will be a higher probability for the occurrence of $\mathrm{Sn}$-rich, $\mathrm{Sn}_{m}-\mathrm{V}$, and $\mathrm{Sn}_{m} \mathrm{P}_{n}-\mathrm{V}$ complexes. In essence that more $\mathrm{Sn}$ atoms could passivate the free vacancy concentration and eventually less number of $\mathrm{P}$ atoms are present around the vacancies can contribute toward the conduction in $\mathrm{Ge}_{1-x} \mathrm{Sn}_{x}$ layers. This assumption holds true, provided the concentration of vacancies remains constant in the layers. Meaning, the incorporation of vacancies during the epitaxial growth would not be affected by the Sn-doping. However, due to the limited solubility ${ }^{27}$ of $\mathrm{Sn}$ in Ge, it becomes extremely challenging to incorporate such high substitutional Sn concentrations. The metastability of Sn-rich epitaxial Ge films also possesses a high risk of Sn precipitation and the degradation of the material properties during post epi processing in device flows. Alternatively, 
the point defect engineering strategies such as codoping with As or Sb (Ref. 17) might enable an increased $\mathrm{P}$ activation in Ge, since the vacancies have a higher attraction for both $\mathrm{As}$ and $\mathrm{Sb}$ atoms (due to stronger binding energy values as shown in Fig. 1) in comparison to the $\mathrm{P}$ atoms. In this case, the majority of vacancies would be passivated by As (or Sb) atoms, provided that the concentration of the vacancies decreases or remains independent of As (or Sb)-doping.

\section{ACKNOWLEDGMENTS}

The imec core CMOS program members, European Commission, the TAKEMI5 ECSEL project, local authorities, and the imec pilot line are acknowledged for their support. A.V. acknowledges long stay abroad grant from the Research Foundation-Flanders (Application No. V410518N). I.M. acknowledges financial support from the Academy of Finland (Project Nos. 285809, 293932, and 319178). CSC-IT Center for Science, Finland is acknowledged for providing the computational resources.

\section{REFERENCES}

${ }^{1}$ G. Impellizzeri, S. Boninelli, F. Priolo, E. Napolitani, C. Spinella, A. Chroneos, and H. Bracht, J. Appl. Phys. 109, 113527 (2011).

${ }^{2}$ A. Chroneos, R. W. Grimes, and H. Bracht, J. Appl. Phys. 106, 063707 (2009).

${ }^{3} \mathrm{H}$. Tahini, A. Chroneos, R. W. Grimes, U. Schwingenschlögl, and H. Bracht, Appl. Phys. Lett. 99, 072112 (2011).

${ }^{4}$ H. A. Tahini, A. Chroneos, R. W. Grimes, U. Schwingenschlögl, and H. Bracht, Phys. Chem. Chem. Phys. 15, 367 (2013).

5J. Vanhellemont and E. Simoen, Mater. Sci. Semicond. Process. 15, 642 (2012).
${ }^{6}$ R. Loo, B. Vincent, F. Gencarelli, C. Merckling, A. Kumar, G. Eneman, L. Witters, W. Vandervorst, M. Caymax, M. M. Heyns, and A. Thean, ECS J. Solid State Sci. Technol. 2(1), N35 (2013).

${ }^{7}$ G. Kresse and J. Furthmüller, Phys. Rev. B 54, 11169 (1996).

${ }^{8}$ G. Kresse and J. Furthmüller, Comput. Mater. Sci. 6, 15 (1996).

${ }^{9}$ P. E. Blöchl, Phys. Rev. B 50, 17953 (1994).

${ }^{10} \mathrm{G}$. Kresse and D. Joubert, Phys. Rev. B 59, 1758 (1999).

${ }^{11}$ J. Heyd, G. E. Scuseria, and M. Ernzerhof, J. Chem. Phys. 118, 8207 (2003).

${ }^{12}$ M. J. Puska and R. M. Nieminen, Rev. Mod. Phys. 66, 841 (1994).

${ }^{13}$ E. Boroński and R. M. Nieminen, Phys. Rev. B 34, 3820 (1986).

${ }^{14}$ I. Makkonen, M. Hakala, and M. J. Puska, Phys. Rev. B 73, 035103 (2006).

${ }^{15}$ M. Alatalo, B. Barbiellini, M. Hakala, H. Kauppinen, T. Korhonen, M. J. Puska, K. Saarinen, P. Hautojärvi, and R. M. Nieminen, Phys. Rev. B 54, 2397 (1996).

${ }^{16}$ I. Makkonen, M. Hakala, and M. J. Puska, J. Phys. Chem. Solids 66, 1128 (2005).

${ }^{17}$ A. Vohra, A. Khanam, J. Slotte, I. Makkonen, G. Pourtois, R. Loo, and W. Vandervorst, J. Appl. Phys. 125, 025701 (2019).

${ }^{18}$ R. Loo, Y. Shimura, S. Ike, A. Vohra, T. Stoica, D. Stange, D. Buca, D. Kohen, J. Margetis, and J. Tolle, Semicond. Sci. Technol. 33, 114010 (2018).

${ }^{19}$ Y. Shimura, S. A. Srinivasan, D. V. Thourhout, R. V. Deun, M. Pantouvaki, J. V. Campenhout, and R. Loo, Thin Solid Films 602, 56 (2016).

${ }^{20}$ J. R. MacDonald, K. Lynn, R. Boie, and M. Robbins, Nucl. Instrum. Methods 153, 189 (1978).

${ }^{21}$ F. Tuomisto and I. Makkonen, Rev. Mod. Phys. 85, 1583 (2013).

${ }^{22}$ P. J. Schultz and K. G. Lynn, Rev. Mod. Phys. 60, 701 (1988).

${ }^{23}$ G. C. Aers, J. Appl. Phys. 76, 1622 (1994).

${ }^{24}$ S. Valkealahti and R. M. Nieminen, Appl. Phys. A 32, 95 (1983).

${ }^{25}$ J. Slotte, S. Kilpeläinen, F. Tuomisto, J. Räisänen, and A. N. Larsen, Phys. Rev. B 83, 235212 (2011).

${ }^{26}$ T. Kalliovaara, J. Slotte, I. Makkonen, J. Kujala, F. Tuomisto, R. Milazzo, G. Impellizzeri, G. Fortunato, and E. Napolitani, Appl. Phys. Lett. 109, 182107 (2016).

27J. P. Fleurial and A. Borshchevsky, J. Electrochem. Soc. 135(9), 2928 (1990). 